UNIVERSIDADE DE SÃO PAULO

FACULDADE DE ODONTOLOGIA DE RIBEIRÃO PRETO

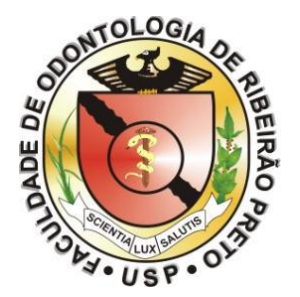

Gabriela Ferian Molina

\title{
Deposição de chumbo no esmalte dentário bovino durante o processo de formação de cárie in vitro
}

Ribeirão Preto 


\section{Gabriela Ferian Molina}

\section{Deposição de chumbo no esmalte dentário \\ bovino durante o processo de formação de}

cárie in vitro

Dissertação apresentada à Faculdade de Odontologia de Ribeirão Preto da Universidade de São Paulo, para a obtenção do título de Mestre, pelo curso de Pós-Graduação no Programa de Reabilitação Oral.

Área de Concentração: Biologia Oral

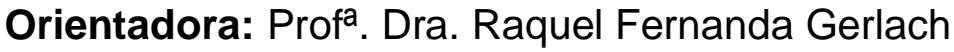

VERSÃO CORRIGIDA

Ribeirão Preto 
AUTORIZO A REPRODUÇÃO E DIVULGAÇÃO DO TEOR TOTAL OU PARCIAL DESTE TRABALHO POR QUALQUER MEIO CONVENCIONAL OU ELETRÔNICO, PARA FINS DE ESTUDO E PESQUISA, DESDE QUE CITADA A FONTE.

Molina, Gabriela Ferian

Deposição de chumbo no esmalte dentário bovino durante o processo de formação de cárie in vitro. Ribeirão Preto, 2012.

41 p. : il. ; $30 \mathrm{~cm}$.

Versão Corrigida da Dissertação de Mestrado, apresentada à Faculdade de Odontologia de Ribeirão Preto/USP. Área de concentração: Biologia Oral. A versão original se encontra disponível na Biblioteca da Unidade Sede do Programa.

1. Esmalte. 2. Chumbo. 3. Microfluorescência de Raio-X por Luz Sincrotron. 4. Dente. 5. Cárie. 
Gabriela Ferian Molina

Dissertação apresentada à Faculdade de Odontologia de Ribeirão Preto da Universidade de São Paulo, para a obtenção do título de Mestre, pelo curso de Pós-Graduação no Programa de Reabilitação Oral.

Área de Concentração: Biologia Oral

Aprovada em:

Banca Examinadora:

1) Prof.(a). Dr.(a).:

Instituição:

Julgamento:

Assinatura:

2) Prof.(a). Dr.(a).:

Instituição:

Julgamento:

Assinatura:

3) Prof.(a). Dr.(a).:

Instituição:

Julgamento:

Assinatura: 


\section{DEDICATÓRIA}

Dedico este trabalho,

A Deus, por iluminar meu caminho durante esta jornada, me dar força e sabedoria para enfrentar os momentos difíceis, saúde e proteção durante esta fase de aprendizagem.

Ao meu pai, Ivo Rubens Molina (in memoriam) por todo seu esforço durante a sua vida, para que eu pudesse estudar e estar aqui hoje, e por ser um exemplo de caráter e bondade.

À minha mãe, Vera Ap. Ferian Molina, por estar sempre ao meu lado me aconselhando, ajudando a enfrentar as dificuldades e sempre torcendo por mim.

À minha avó, Laura Ribeiro Molina, por ser um exemplo de força e vida, pelo seu carinho, amor e torcida durante toda a minha vida.

Aos meus irmãos, Paula e Fábio, por estarem comigo durante todos os momentos.

Aos meus sobrinhos, Ana Carolina, João Pedro e Letícia, por me fazerem sorrir e me divertir sempre.

Muito Obrigada meus queridos, é com muito carinho que eu dedico a vocês esta conquista! 


\section{AGRADECIMENTOS}

Em especial, à minha orientadora, Professora Raquel Fernanda Gerlach, pelo carinho, paciência e atenção durante esses dois anos de mestrado e também durante os dois anos de iniciação científica, pelos seus ensinamentos e seu entusiasmo em ensinar seus alunos, pelos sonhos realizados que não teriam sido concretizados sem a sua ajuda.

À Glauce Regina Costa de Almeida, por estar comigo desde o início da minha jornada, por sua contribuição neste trabalho, por seus ensinamentos, pelo seu carinho, paciência e por sua amizade.

À Prof ${ }^{\mathrm{a}} \mathrm{Dr}^{\mathrm{a}}$ Maria Cristina Borsatto, por ceder seu laboratório, para realização de alguns experimentos.

À Carolina de Souza Gerra, pela ajuda na análise das amostras no Laboratório Nacional de Luz Sincrotron (LNLS), em Campinas, pelo seu carinho e amizade.

À Junia Ramos, por sua companhia e amizade.

Aos professores da banca, pelo tempo e atenção dispensados durante a leitura deste trabalho.

Aos amigos de turma, Olívia, Juliana, Leonardo e Cristiane, pela companhia, amizade e carinho. Em especial à Cris por todo seu carinho, por estar sempre junto a mim, me incentivando e me apoiando.

Aos amigos de laboratório, Luciana, Alejandro, Aline, César, Marcus e Felipe, pela ajuda, companhia e por tornarem o ambiente de trabalho tão agradável.

Ao meu amigo José Raul, por fazer parte da minha vida, por estar comigo durante esse último ano, me apoiando e sendo a melhor companhia em todos os momentos.

Às minhas velhas e novas amigas Danielle, Mariana, Ariany, Lívia, Amanda, Aline, Graziela, Karina, Ana Beatriz, Glauce, Lourdes, Vanessa, Tatiana, Ana Carolina e Izabela pelo apoio e torcida e também por serem pessoas tão queridas.

À minha grande amiga e irmã Christiane Peccinatti por todo seu apoio, carinho, paciência, incentivo e por fazer parte da minha vida.

À FAPESP por todo seu apoio para que este trabalho pudesse ser concluído. 


\section{EPÍGRAFE}

"Feliz aquele que transfere o que sabe e aprende o que ensina."

Cora Coralina 
MOLINA, G. F. Deposição de chumbo no esmalte dentário bovino durante o processo de formação de cárie in vitro. Ribeirão Preto, 2012. 41p. Dissertação (Mestrado em Biologia Oral). Faculdade de Odontologia de Ribeirão Preto, Universidade de São Paulo.

\section{RESUMO}

Assim como o flúor, o chumbo se acumula sobre a superfície do esmalte de dentes não irrompidos , o que ainda não se sabe, é se durante o processo de formação da cárie dentária, ele também pode se acumular sobre o esmalte. Este estudo avalia a distribuição espacial do chumbo em blocos dentários bovino submetidos a um regime de ciclagens de $\mathrm{pH}$ simulando o processo de desenvolvimento da cárie dentária. Os blocos de esmalte dentário foram submetidos a oito ciclos de desmineralização e remineralização, sendo que, na solução correspondente ao grupo experimental 1 (E1), foram adicionados $30 \mu \mathrm{g} / \mathrm{l}$ de acetato de chumbo e na solução correspondente ao grupo experimental 2 (E2), foram adicionados $300 \mu \mathrm{g} / \mathrm{l}$ de acetato de chumbo, enquanto que, na solução correspondente ao grupo controle (C) não foi adicionado chumbo. Após os ciclos de desmineralização e remineralização, foram confeccionadas, a partir dos blocos dentários, fatias de $100 \mu \mathrm{m}$ de espessura. Essas fatias foram analisadas por microscopia de luz polarizada para observar a extensão da lesão cariosa formada e também foram levadas para análise através da microfluorescência de raio-x por luz Sincrotron. As lesões de cárie foram observadas ao longo de toda a superfície do esmalte apresentando uma extensão de aproximadamente $120 \mu \mathrm{m}$. Foi observado no esmalte, um gradiente de concentração de chumbo que diminuía da superfície em direção à dentina. Os sinais mais altos de chumbo foram encontrados no grupo E2. E as diferenças estatisticamente significantes, foram observadas na profundidade de esmalte 0 (superfície do esmalte) na comparação entre o grupo C e o grupo E2 (C vs $\mathrm{E} 2 ; \mathrm{p}=0,029$ ) e na profundidade de esmalte de $50 \mu \mathrm{m}$, nas comparações entre o grupo $C$ e grupo E2 (C vs E2; $p=0,029)$ e entre o grupo E1 e o grupo E2 ( $E 1$ vs $E 2 ; p=$ $0,029)$. Assim, este estudo sugere que se o chumbo estiver presente na cavidade oral, durante o processo de desenvolvimento da lesão cariosa, ele pode se acumular ao esmalte dentário.

Palavras-Chaves: Esmalte, Chumbo, Microfluorescência de Raio-X por Luz Sincrotron, Dente, Cárie. 
MOLINA, G. F. Deposição de chumbo no esmalte dentário bovino durante o processo de formação de cárie in vitro. Ribeirão Preto, 2012. 41p. Dissertação (Mestrado em Biologia Oral). Faculdade de Odontologia de Ribeirão Preto, Universidade de São Paulo.

\begin{abstract}
Like fluoride, lead $(\mathrm{Pb})$ accumulates on the enamel surface pre-eruptively, but it is not yet known whether it also deposits on enamel while dental caries is developing. This study evaluates $\mathrm{Pb}$ distribution in bovine enamel slabs submitted to a $\mathrm{pH}$-cycling regimen simulating the caries process. The slabs were subjected to 8 cycles of deand remineralizing conditions, and $\mathrm{Pb}$ (as acetate salt) was added to the de- and remineralized solutions at concentrations of $30 \mu \mathrm{g} / \mathrm{l}$ (experimental group, E1) and 300 $\mu \mathrm{g} / \mathrm{l}$ (experimental group, E2). The control group (C) consisted of solutions to which $\mathrm{Pb}$ was not added. After the $\mathrm{pH}$ cycling, $100 \mu \mathrm{m}$ sections of the slabs were analyzed by polarizing microscopy, to observe the extent of caries-like lesions, and these sections were used for $\mathrm{Pb}$ estimation by Synchrotron radiation X-ray microfluorescence. Caries lesions were observed along all superficial enamel surfaces to an extent of $120 \mu \mathrm{m}$. A Pb concentration gradient was observed in enamel, which decreased toward dentine. The highest Pb signals were observed for group E2, and the differences were statistically significant at enamel depths of 0 (C vs. $E 2 ; p=0.029)$ and $50 \mu \mathrm{m}(\mathrm{C}$ vs. $\mathrm{E} 2$ and $\mathrm{E} 1 \mathrm{vs}$. $\mathrm{E} 2 ; \mathrm{p}=0.029)$. In conclusion, this study suggests that if $\mathrm{Pb}$ is present in the oral environment, it may deposit in enamel during the caries process.
\end{abstract}

Keywords: Enamel, Lead, Synchrotron radiation X-ray microfluorescence, Teeth, Caries. 


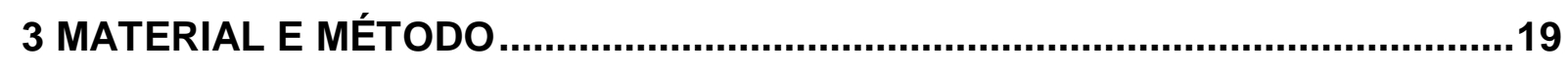

3.1 Preparação dos blocos de esmalte …….................................................... 19

3.2 Preparo das soluções desmineralizante e remineralizante ............................20

3.3 Ciclos de desmineralização e remineralização ...............................................20

3.4 Inclusão dos blocos dentários em resina e análise microscópica .....................21

3.5 Análise de microfluorescência de raio- x por luz Sincrotron ..............................23

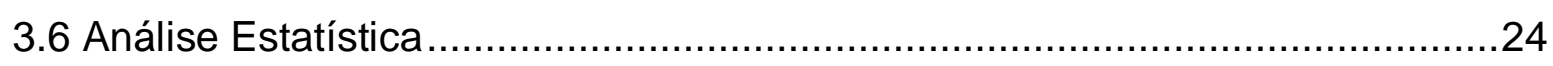

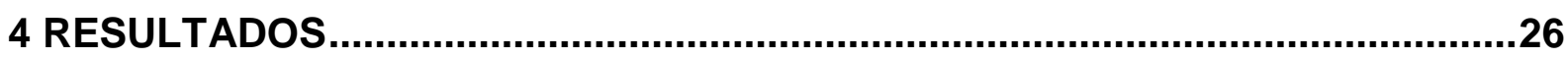

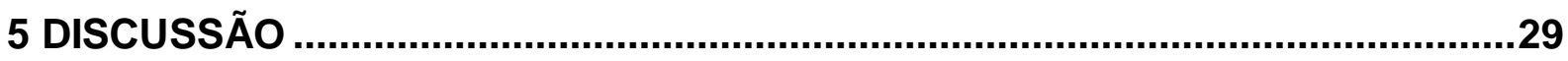

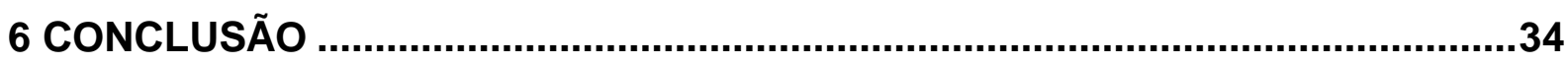

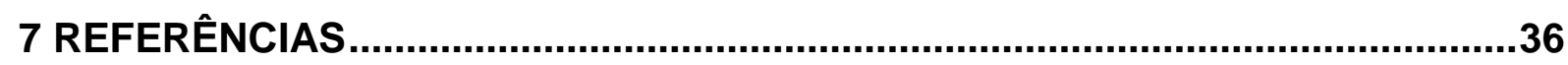

ANEXO 


\section{INTRODUÇÃO}

O esmalte dentário é único entre os tecidos mineralizados, devido ao seu alto teor mineral (Simmer e Hu, 2001), sendo a hidroxiapatita o componente mais abundante. Ele é composto por cristalitos de esmalte altamente organizados e hermeticamente embalados, que compreendem $87 \%$ do seu volume e $95 \%$ do seu peso (Nanci, 2008). Enquanto outros tecidos mineralizados possuem aproximadamente $20 \%$ de material orgânico, o esmalte maduro apresenta menos de $1 \%$ desse material. Os cristalitos de esmalte possuem mais de mil vezes o volume dos cristais correspondentes no osso, dentina e cemento (Simmer e Hu, 2001).

A mineralização do esmalte é um processo que dura um longo período de tempo: desde os estágios iniciais de secreção da matriz até o irrompimento do dente na cavidade bucal (Smith, 1998). A maioria dos minerais é depositada sobre a matriz de esmalte, durante a fase de maturação. Portanto, qualquer outro íon presente no corpo do indivíduo durante essa fase, pode se acumular ao esmalte que está sendo formado (Simmer e Hu, 2001). Quando se trata da estrutura morfológica do esmalte, esta não é modificada depois de formada e, consequentemente, as alterações adquiridas em sua estrutura durante seu processo de formação serão marcas permanentes (Gerlach et al., 2002). Mas eventos como o processo de desmineralização e remineralização ainda podem provocar mudanças no conteúdo mineral do esmalte dentário. Essa informação é relevante quando há a possibilidade de utilizar o esmalte dentário como um biomarcador, para detectar elementos que se acumulam nos tecidos mineralizados. Substâncias externas, como o chumbo, podem se acumular no esmalte dentário durante a sua formação, e a análise desse 
tecido nos permite então identificar o grau de exposição do indivíduo a tal substância.

O chumbo é um metal pesado com inúmeras propriedades, possui baixo ponto de fusão e é altamente maleável, devido a estas características, foi amplamente utilizado para diversos fins, tanto pelas antigas civilizações como pelas sociedades modernas (Grant, 2009). O intenso uso desse metal pelas sociedades modernas, no sistema de distribuição de água, como aditivo para tintas e gasolina, para aplicações eletrônicas, entre outros, causou um grande aumento da exposição a chumbo nas populações humanas em todo o mundo, principalmente durante o século vinte. No entanto, apesar de ser um material de enorme utilidade, ele também foi reconhecido por ser intensamente tóxico quando exposto a altas doses (Grant, 2009).

As primeiras preocupações com relação à toxicidade do chumbo e os danos que esse elemento poderia causar à saúde surgiram por volta do início do século 20, quando médicos, primeiramente na Austrália e depois nos Estados Unidos, começaram a diagnosticar casos de intoxicação por chumbo em crianças, esses casos geralmente eram associados ao chumbo presente tanto nas tinturas de parede quanto nas tinturas de brinquedos (Rosner et al., 2005). Segundo esses autores, naquela época, sintomas de intoxicação por chumbo, como convulsões, eram simplesmente explicados como resultado de um trauma físico durante o nascimento ou como uma infecção bacteriana do sistema nervoso central, ou seja, não se pensava que pudesse ter alguma relação com chumbo. Segundo Cleymaet et al. (1991e), a intoxicação por chumbo em humanos pode levar a disfunções como retardo mental, paralisia cerebral e hiperatividade. Em 1991, o Centro de Controle e Prevenção de Doenças (CDC) nos Estados Unidos determinou que o nível de 
chumbo no sangue das crianças com necessidade de intervenção seria de $15 \mu \mathrm{g} / \mathrm{dL}$ e também determinou que fossem implantadas atividades de prevenção contra a intoxicação por chumbo em toda comunidade onde as crianças apresentassem níveis de chumbo no sangue acima de 10 $\mathrm{g} / \mathrm{dL}$ (ACCLPP, 2012).

Várias são as fontes de exposição humana a chumbo, no Brasil, ele pode estar presente nos alimentos, em tinturas para cabelo, tintas em brinquedos, bebidas alcoólicas, utensílios de PVC, sem falar das fontes industriais como a indústria da borracha e a fabricação de baterias (Capitani et al., 2009).

A contaminação do homem pelo chumbo se dá através dos alimentos, água, ar urbano de cidades industrializadas e/ou em países onde a gasolina aditivada com chumbo ainda é utilizada, tintas e solo contaminado em áreas peri-fabris (Capitani, 2009).

Em 1979, Needleman et al., observaram que crianças que apresentavam altos níveis de chumbo na dentina, eram mais dispersas dentro da sala de aula, tinham mais sono durante o dia, apresentavam dificuldades em seguir orientações e também eram mais desorganizadas, comparadas as outras crianças. No entanto, outro estudo mostrou que em níveis baixos de exposição, o chumbo também pode ter efeitos neurotóxicos sobre as crianças (Needleman et al., 1990).

Vários estudos têm mostrado que o esmalte dentário é conhecido por acumular altas quantidades de chumbo sobre sua superfície (Cleymaet et al., 1991a; Cleymaet et al., 1991b; Cleymaet et al., 1991c; Cleymaet et al., 1991d; Gomes et al., 2004; Costa de Almeida et al., 2007). Essa característica faz desse tecido um importante biomarcador de exposição a esse elemento. Além disso, muitos estudos mostram que existe uma relação entre a exposição a chumbo do meio ambiente e o chumbo presente na superfície do esmalte de dentes permanentes (Cleymaet et al., 
1991a; Cleymaet et al., 1991b; Cleymaet et al., 1991c; Cleymaet et al., 1991d) e de dentes decíduos (Gomes et al., 2004; Costa de Almeida et al., 2007; de Almeida et al., 2008). Segundo esses estudos, os níveis de concentração de chumbo na superfície do esmalte dentário se assemelham aos níveis de chumbo encontrados no ambiente onde essas pessoas vivem. Uma correlação positiva foi observada (Costa de Almeida et al., 2011) quando o chumbo presente na superfície do esmalte dentário decíduo foi comparado ao chumbo encontrado na superfície do esmalte de dentes permanentes, com relação ao chumbo presente no meio ambiente (Costa de Almeida et al., 2010).

O comportamento da concentração de chumbo no esmalte dentário assemelha-se ao comportamento da concentração de flúor, ou seja, ocorre uma diminuição dessa concentração a partir da superfície do esmalte para as regiões mais profundas do dente (Weatherell et al., 1973). Esta característica foi descrita tanto para dentes humanos permanentes (Brudevold and Steadman, 1956; Brudevold et al., 1977; Purchase and Fergusson, 1986; Arora et al., 2005) como para dentes decíduos (Gomes et al., 2004; Costa de Almeida et al., 2007).

Em estudo anterior, de Almeida et al. (2008), descreveu um platô sobre a quantidade de chumbo na superfície do esmalte de dentes decíduos de crianças que viviam em áreas cuja contaminação por chumbo era bem conhecida, mostrando que os valores médios de chumbo no esmalte dentário das crianças que viviam nas áreas contaminadas, obtidos através de biópsias de esmalte bem profundas eram próximos de $600 \mu \mathrm{g} / \mathrm{g}$ de chumbo, enquanto que, no esmalte dentário das crianças que viviam em áreas aparentemente não contaminadas os valores médios de chumbo no esmalte diminuíam de 278 para $121 \mu \mathrm{g} / \mathrm{g}$. Também foi investigado a exposição a chumbo em crianças com idade entre 4 e 8 anos, através da análise da 
superfície do esmalte dentário (Gomes et al., 2004; Costa de Almeida et al., 2007; de Almeida et al., 2008; Costa de Almeida et al., 2009), no entanto, com relação ao processo de formação da cárie, e como ele poderia influenciar na incorporação de chumbo ao esmalte, nada foi estudado até agora.

O processo de formação da cárie envolve a desmineralização e remineralização do esmalte dentário, porém, o que não se sabe é se o chumbo presente nas soluções que banham o esmalte durante a formação da lesão cariosa poderia ser incorporado a ele. Sendo também importante saber até qual profundidade ele seria incorporado.

Assim, diante do exposto acima, seria importante determinar, se durante 0 processo de desmineralização e remineralização in vitro, o chumbo é capaz de se incorporar ao esmalte dentário, sendo esta hipótese confirmada, também seria importante analisar até qual profundidade do esmalte o chumbo poderia ser incorporado. 
2 PROPOSIÇÃO 


\section{PROPOSIÇÃO}

2.1 Determinar a distribuição espacial de chumbo em blocos de esmalte de dentes bovino, submetidos a ciclos de desmineralização e remineralização in vitro;

2.2 Se a incorporação de chumbo no esmalte durante o processo de desmineralização e remineralização in vitro, for confirmada, analisar até qual profundidade o chumbo pode ser encontrado. 
3 MATERIAL E MÉTODO 


\section{MATERIAL E MÉTODO}

\subsection{Preparação dos blocos de esmalte}

Para este estudo foram utilizados 15 blocos de esmalte dentário, que foram confeccionados a partir da coroa dentária de dentes bovinos. Como os dentes foram conseguidos em frigoríficos, não houve a necessidade de aprovação desse estudo pelo Comitê de Ética da instituição, na qual a pesquisa foi realizada.

Primeiramente a coroa dentária foi separada da raiz, utilizando-se um disco diamantado em uma cortadeira, para posterior confecção dos blocos dentários. As amostras foram limpas com solução de Triton X-100 a (0,1\%) e escova dental, em seguida, foram submetidas a três ciclos de lavagens em ultra-som com água ultrapura (milli $\mathrm{Q}$ ), durante 10 minutos cada, e então foram secas à temperatura ambiente por $18 \mathrm{~h}$, dentro de uma capela de fluxo laminar.

Os blocos de esmalte ( $2 \mathrm{~mm} \times 4 \mathrm{~mm}$ ) foram confeccionados (Cury et al., 2000). Uma fita adesiva foi colocada sobre o esmalte vestibular de cada bloco e as demais superfícies foram isoladas com adesivo dentinário e resina flow. Ao final, a fita adesiva foi removida da superfície do esmalte vestibular, para que toda a área do esmalte pudesse ficar exposta (em contato) com as soluções desmineralizantes e remineralizantes, durante os ciclos de desmineralização e remineralização.

Os blocos de esmalte foram divididos aleatoriamente em três grupos: grupo controle "C" ( $n=5)$, grupo experimental 1 "E1" (n=5) e grupo experimental 2 "E2" $(n=5)$. 


\subsection{Preparo das soluções desmineralizante e remineralizante}

As soluções foram preparadas de acordo com o método descrito por Queiroz et al. (2008).

Solução desmineralizante: tampão acetato $0,05 \mathrm{~mol} / \mathrm{l}, \mathrm{pH}$ 5,0 contendo 1,28 $\mathrm{mmol} / \mathrm{l} \mathrm{Ca} ; 0,74 \mathrm{mmol} / \mathrm{PO} \mathrm{PO}_{4}$ e $0,03 \mu \mathrm{g} / \mathrm{ml}$ preparadas a partir dos sais $\mathrm{Ca}\left(\mathrm{NO}_{3}\right)_{4}$. $4 \mathrm{H}_{2} \mathrm{O} ; \mathrm{KH}_{2} \mathrm{PO}_{4}$, e NaF, respectivamente.

Solução remineralizante: tampão Tris $0,1 \mathrm{~mol} / \mathrm{l}, \mathrm{pH} 7,0$ contendo $1,5 \mathrm{mmol} / \mathrm{l}$ Ca, 0,9 mmol// $\mathrm{PO}_{4}, 150 \mathrm{mmol} / \mathrm{l} \mathrm{KCl}$, e 0,05 $\mu \mathrm{g} \mathrm{F/ml.}$

A quantidade de solução desmineralizante e remineralizante utilizada em cada recipiente foi de $6,25 \mathrm{ml} / \mathrm{mm}^{2}$ de esmalte e $3,12 \mathrm{ml} / \mathrm{mm}^{2}$ de esmalte, respectivamente.

\subsection{Ciclos de desmineralização e remineralização}

Os ciclos de desmineralização e remineralização tiveram duração de oito dias. Diariamente os blocos dentários eram colocados na solução desmineralizante durante quatro horas, e em seguida, eram colocados na solução remineralizante por mais vinte horas e mantidos em ambiente com temperatura de $37^{\circ} \mathrm{C}$. Sempre que os blocos passavam de uma solução para outra, eles eram colocados em água deionizada durante cinco minutos sob agitação. No quarto dia de experimento as soluções de desmineralização e remineralização foram substituídas por soluções recém-preparadas. Ao final do período de ciclagem, os blocos ainda foram mantidos na solução de remineralização por mais $24 \mathrm{~h}$ (Argenta et al., 2003). 
A solução remineralizante era diferente para os três grupos. Para o grupo $\mathrm{C}$, a solução era composta de $300 \mu \mathrm{g} / \mathrm{l}$ de acetato de sódio, para o grupo E1, era composta de $30 \mu \mathrm{g} / \mathrm{l}$ de acetato de chumbo e para o grupo E2, era composta de 300 $\mu \mathrm{g} / \mathrm{l}$ de acetato de chumbo. Estas concentrações foram baseadas nos achados de Costa de Ameida et al. (2009), que observou uma concentração máxima de chumbo de $36 \mu \mathrm{g} / \mathrm{l}$ na saliva de crianças que viviam em área já conhecida pela contaminação por chumbo.

\subsection{Inclusão dos blocos dentários em resina e análise microscópica}

Os blocos dentários de cada grupo foram incluídos em resina autopolimerizável. Após a polimerização da resina, com auxílio de um disco diamantado e uma cortadeira, uma fatia de aproximadamente $300 \mu \mathrm{m}$ de espessura foi removida de cada grupo. O corte foi feito no sentido longitudinal, ou seja, o disco era posicionado perpendicularmente ao centro do esmalte de cada bloco e seguia em direção à dentina. Uma das fatias é representada pela figura 1. Em seguida, essas fatias foram polidas com lixas d'água de granulação decrescente e água ultrapura (milli Q) até que a espessura de $100 \mu \mathrm{m}$ fosse atingida. Depois de polidos, os cortes foram levados para análise em microscópio de luz polarizada (Axioskop 40, Carl Zeiss), com utilização do filtro de retardo vermelho I. O ar atmosférico foi o meio utilizado para a observação da profundidade da lesão de cárie formada. As imagens foram capturadas pelo software Axio Vision LE 4.7.

As lesões cariosas apresentaram áreas com aumento de porosidade e apareceram na imagem como zonas escuras no esmalte, quando foram analisadas por microscopia de luz polarizada (figura 2). 


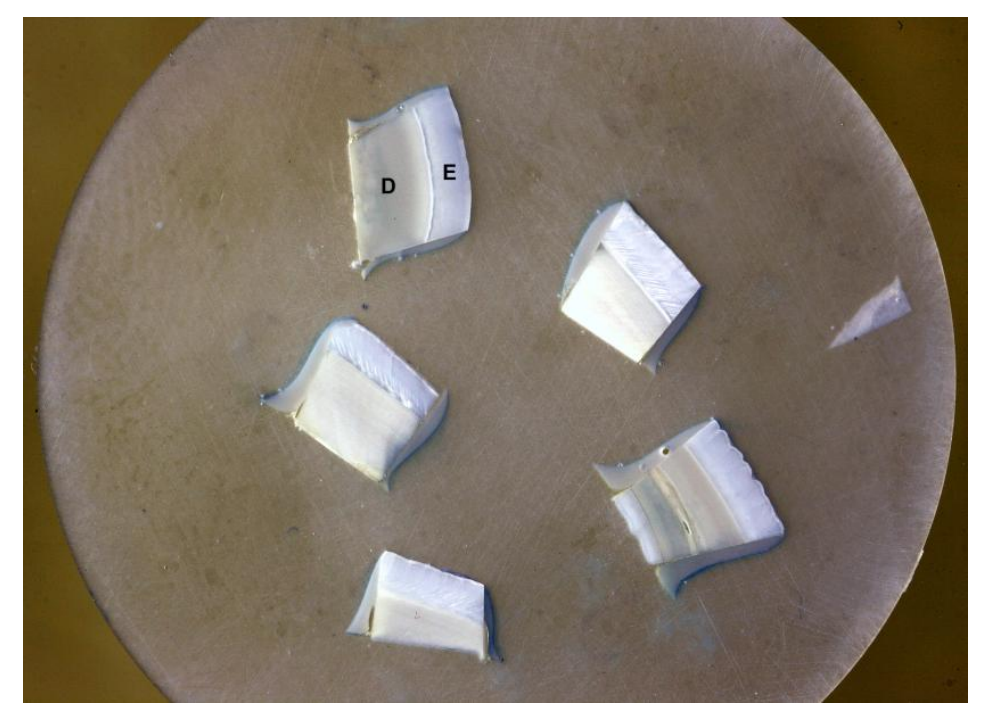

Figura 1: A imagem representa uma das fatias dos blocos dentários bovino que foram incluídos em resina, para posterior análise por microscopia de luz polarizada. 'D' está indicando a dentina e 'E' está indicando o esmalte dentário. A imagem foi feita com câmera digital Canon EOS Rebel XTi, com distância focal de $177 \mathrm{~mm}$ e tempo de exposição de $6 \mathrm{~s}$.

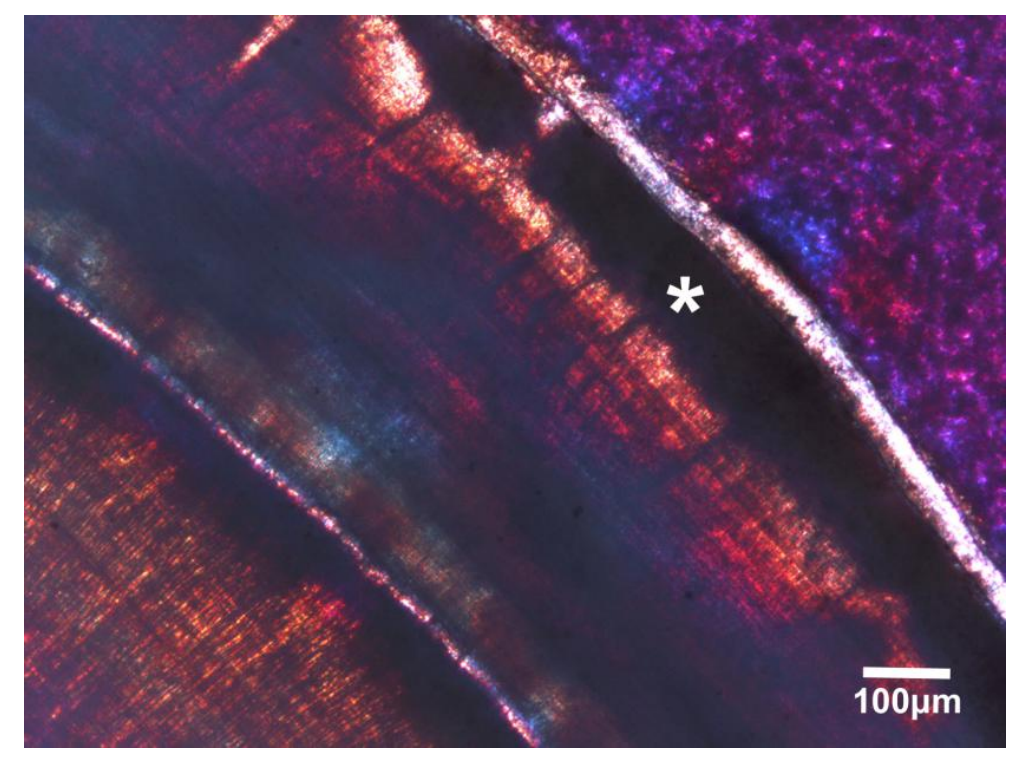

Figura 2: Fotografia da superfície do esmalte dentário sob microscopia de luz polarizada. A lesão cariosa aparece sobre a superfície do esmalte como área escura e está indicada pelo asterisco. 


\subsection{Análise de microfluorescência de raio- x por luz Sincrotron}

A distribuição espacial de chumbo foi determinada pela análise dos blocos através da Microfluorescência de Raio-X estimulados por Luz Sincrotron, no Laboratório Nacional de Luz Sincrotron (LNLS), em Campinas, São Paulo.

O chumbo foi medido na superfície do esmalte (ponto 0), e em outros cinco pontos a partir da sua superfície: $50 \mu \mathrm{m}, 100 \mu \mathrm{m}, 150 \mu \mathrm{m}, 200 \mu \mathrm{m}$ e $500 \mu \mathrm{m}$. A composição elementar foi determinada usando micro feixes SR de raio-X fluorescente. As medidas foram realizadas em geometria padrão $\left(45^{\circ}+45^{\circ}\right)$.

As amostras foram excitadas com feixes de luz branca, que são focados com capilar cônico com 20 micrometros de diâmetro. A imagem da amostra projetada no visor (magnitude 500x) foi usada para definição dos pontos a serem analisados na amostra. O espectro fluorescente foi registrado com um detector Si (Li) de 165 eV FWHM a $5.9 \mathrm{keV}$ em ar atmosférico posicionado a $90^{\circ}$ da direção de incidência. As leituras lineares automáticas foram feitas com as amostras posicionadas no plano da imagem com acurácia de $0,5 \mu \mathrm{m}$ nos três eixos ( $x, y$ e $z$ ). O padrão de leitura adotado foi semelhante para todas as amostras. Os espectros de raio-X foram obtidos em diferentes profundidades do esmalte, ou seja, na superfície do esmalte, e nas profundidades de 50, 100, 150, 200 e $500 \mu \mathrm{m}$. Cada região da amostra analisada era excitada durante 300 s, e os espectros de raio- $X$ adquiridos foram avaliados pelo programa, Sistema de Análise Quantitativa de Raio-X (QXAS), distribuído pela Agência Internacional de Energia Atômica, a fim de obter a intensidade de raio- $\mathrm{X}$ ideal para cada elemento. 


\subsection{Análise Estatística}

A intensidade relativa de fluorescência de distribuição do chumbo foi analisada quanto à normalidade, e a distribuição foi não-normal. O teste KruskalWallis foi usado para comparar as intensidades relativas (que expressam chumbo) nas diferentes profundidades. Este teste também foi utilizado para verificar se as diferenças vistas entre os três grupos (C, E1e E2) eram estatisticamente significantes em cada profundidade. Quando as diferenças eram estatisticamente significantes, foi feita a comparação entre cada par (3 pares) de grupos para uma mesma profundidade, sendo neste caso utilizado o teste de Mann-Whitney. As diferenças foram consideradas estatisticamente significantes ao nível de 5\%. 


\section{RESULTADOS}

Sinais mais altos de chumbo foram observados para o grupo E2, no entanto, uma comparação dos sinais de chumbo obtidos para os três grupos (C, E1 e E2) revelou uma diferença estatisticamente significante nas profundidades de esmalte 0 ( $C$ versus $E 2, p=0,029$ ); enquanto que, nenhuma diferença foi encontrada entre $C$ versus $E 1(p=0,34)$ ou entre $E 1$ versus $E 2,(p=0,20)$

Também houve diferença estatisticamente significante quando os resultados obtidos na profundidade de esmalte de $50 \mu \mathrm{m}$ foram comparados (C versus E2 e E1 versus $E 2 ; p=0,029)$, enquanto que a comparação entre $C$ versus $E 1$ não atingiu diferença estatisticamente significante $(p=0,34)$.

Na profundidade de $100 \mu \mathrm{m}$, a comparação entre E1 e E2 quase atingiu significância estatística ( $p=0,057)$, sugerindo que existe uma diferença, mas uma amostra maior seria necessária para demonstrá-la. Não foi encontrada diferença entre C e E1 ( $p=0,10)$ ou entre C e E2 $(p=0,22)$.

$\mathrm{Na}$ profundidade de $150 \mu \mathrm{m}$, as diferenças foram ainda mais baixas e, novamente, não alcançou significância estatística, mas o grupo E2 exibiu um sinal de chumbo quatro vezes maior, quando comparado ao grupo controle e ao grupo E1 ( $p=0,34$ para ambas as comparações). 


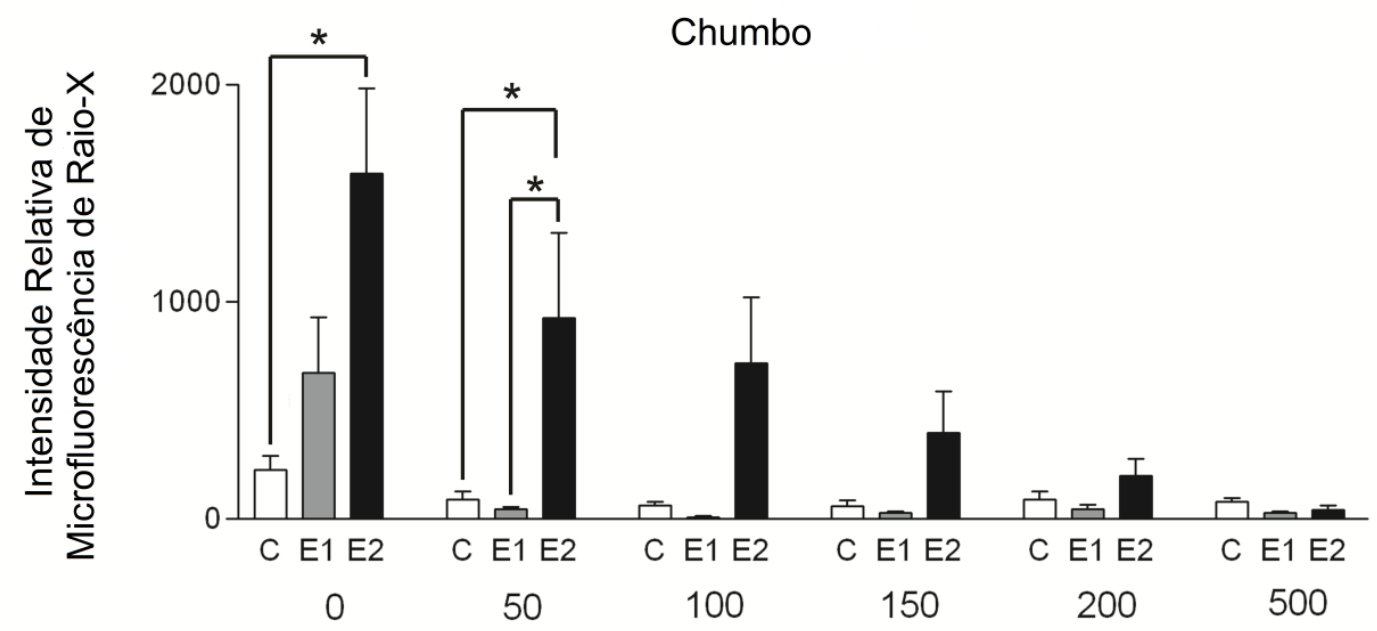

Profundidade de esmalte $(\mu \mathrm{m})$

Figura 3: Distribuição espacial de chumbo após o processo de indução artificial de cárie, C grupo controle; E1 grupo experimental 1 ( $30 \mu \mathrm{g} / \mathrm{L}$ de acetato de chumbo) e E2 grupo experimental 2 (300 $\mu \mathrm{g} / \mathrm{L}$ de acetato de chumbo). ${ }^{*} p<0,05$

Os valores absolutos de chumbo, na profundidade de $500 \mu \mathrm{m}$, apresentaramse baixos em todos os grupos, e nenhuma diferença estatisticamente significante foi observada. Nesta profundidade, não foi observada atividade de cárie através da análise por microscopia de luz polarizada, este fato explica a análise do esmalte até esta profundidade, pois os resultados sugerem uma maior dificuldade de se encontrar chumbo nas camadas mais profundas do esmalte.

A intensidade relativa do chumbo na superfície do esmalte (profundidade de esmalte $=0)$ foi $227,3(\mathrm{DP}= \pm 126,9), 540,0(\mathrm{DP}= \pm 533,4)$ e 1.592,0 $(\mathrm{DP}= \pm 782,6)$ para os grupos C, E1, E2, respectivamente. Na profundidade de esmalte de $50 \mu \mathrm{m}$ a intensidade relativa foi de $91,0 \mathrm{~Pb}(\mathrm{DP}= \pm 72,4), 36,0(\mathrm{DP}= \pm 26,8)$ e $926,0(\mathrm{DP}= \pm$ $786,4)$ para os grupos C, E1 e E2, respectivamente.

A figura 3 também evidencia uma diminuição no sinal de chumbo a partir da superfície do esmalte para as camadas mais profundas, especialmente quando se vai da superfície do esmalte (profundidade de esmalte $=0$ ) para uma profundidade de $50 \mu \mathrm{m}$. Esse gradiente foi mais pronunciado no grupo E2. 


\section{DISCUSSÃO}

Essas grandes diferenças em valores absolutos indicam que seria necessário estudar um maior número de blocos, a fim de obter menor variabilidade nos sinais de chumbo, nas camadas mais profundas do esmalte, evitando assim erro estatístico tipo dois, que pode ocorrer quando o tamanho da amostra empregado é pequeno.

As intensidades relativas de fluorescência dentro do mesmo grupo, em diferentes profundidades do esmalte, também foram comparados (diferenças não são indicados no gráfico). Para os grupos C e E1, a maioria das comparações entre a profundidade de 0 e qualquer outra profundidade alcançou significância estatística $(p<0,05)$ ou valores de $p<0,08$, sugerindo significância estatística. Ainda nesses dois grupos, a intensidade do chumbo foi maior apenas na superfície do esmalte. Assim como para o grupo E2, nenhuma diferenças estatísticamente significante foi alcançada nas comparações entre 0 e $50 \mu \mathrm{m}$ ou entre 0 e $100 \mu \mathrm{m}$, enquanto que nas comparações entre 0 e qualquer outra profundidade alcançou significância estatística $(p<0,05)$.

Com relação a incorporação de chumbo no esmalte, estudos com resolução e sensibilidade suficientes demonstraram claramente que a superfície do esmalte contém maiores quantidades de chumbo em comparação com o esmalte mais profundo ou a dentina (Arora et al., 2005; de Souza Guerra et al., 2010).

Os resultados deste estudo mostram que o processo de cárie permite a incorporação de chumbo no esmalte dentário, tanto no grupo controle (no qual o chumbo não estava presente nas soluções desmineralizantes e remineralizante) como nos grupos experimentais (E1 e E2), e essa incorporação aparentemente 
ocorreu de maneira dose-dependente. Enquanto que no caso do grupo E1 (30 $\mu \mathrm{g} / \mathrm{L}$ de acetato de chumbo) a concentração de chumbo foi maior apenas na superfície do esmalte, no grupo E2 (300 $\mu \mathrm{g} / \mathrm{L}$ de acetato de chumbo), a quantidade de chumbo também aumentou nas camadas mais profundas do esmalte.

Embora não tenha alcançado diferença estatística ao nível de 5\%, aumento nos valores absolutos de chumbo foram observados, mesmo em uma profundidade de $200 \mu \mathrm{m}$ de esmalte, que é o dobro da profundidade do processo de cárie observado sob a luz polarizada. Isso pode indicar que, quando o chumbo está presente em maior concentração, ele penetra no esmalte para distâncias maiores que aquelas observadas como a área escura referente à lesão cariosa.

A diferença entre os resultados obtidos para os grupos E1 e E2 sugere que há um comportamento distinto em termos de incorporação de chumbo no esmalte, dependendo das concentrações desse elemento que são encontradas no líquido que banha o esmalte. Os níveis de chumbo na saliva variam de 0,09 a 9,10 $\mu \mathrm{g} / \mathrm{L}$ e de 1,64 a 36,19 $\mu \mathrm{g} / \mathrm{L}$, valores descritos em populações não contaminadas e contaminadas, respectivamente (Costa de Almeida et al., 2009). Portanto, a concentração de chumbo no grupo E2 é relativamente alta, mesmo quando comparada às concentrações salivares de chumbo detectado em indivíduos expostos. Além disso, se considerar que os níveis de chumbo na saliva assemelham-se aos níveis plasmáticos/séricos de chumbo, os resultados se tornam ainda mais interessantes. Para populações não expostas, os níveis plasmáticos e séricos de chumbo são descritos na faixa de 0,14 a 0,38 $\mu \mathrm{g} / \mathrm{L}$ (Bergdahl et al., 1999) e menos de 0,03 $\mu \mathrm{g} / \mathrm{L}$ (limite de detecção) para 2,6 $\mu \mathrm{g} / \mathrm{L}$ (Costa de Almeida et al., 2010), respectivamente. Para as populações expostas, os níveis plasmáticos de 
chumbo são descritos de 0,02 a 2,50 $\mu \mathrm{g} / \mathrm{L}$ para homens e 0,03 a 1,60 $\mu \mathrm{g} / \mathrm{L}$ para as mulheres (Barbosa et al., 2006).

Os níveis plasmáticos de chumbo são importantes quando o processo de incorporação de chumbo ao esmalte é uma questão de especulação. Isso ocorre porque durante a fase de formação do esmalte, na fase de maturação, em que o conteúdo mineral do esmalte aumenta de $30 \%$ para $96 \%$, acredita-se que a matriz do esmalte fica em contato direto com o plasma, e esta fase pode durar até quatro anos em muitos dentes permanente (Smith, 1998).

Muitos estudos têm mostrado que o chumbo se acumula nos primeiros $10 \mu \mathrm{m}$ de esmalte (Gomes et al., 2004; Arora et al., 2005; Costa de Almeida et al., 2007; de Almeida et al., 2008). Há também evidências de aumento dos níveis de chumbo na saliva de crianças que vivem em áreas com contaminação ambiental por chumbo (Costa de Almeida et al., 2009). No entanto, se o processo de cárie poderia influenciar a incorporação do chumbo (possivelmente a partir da saliva) no esmalte, ainda não tinha sido estudado.

Este estudo demonstra que os níveis mais elevados de chumbo encontrados na saliva de crianças, e que foram semelhantes no caso do grupo E1, irá provavelmente resultar em alterações na superfície do esmalte até $50 \mu \mathrm{m}$ apenas. Com relação a concentração de chumbo empregada para o grupo E2, esta é provavelmente muito maior do que a concentração encontrada na saliva ou no plasma de indivíduos expostos a este elemento.

As mudanças na distribuição de chumbo verificadas no caso do grupo controle e dos grupos experimentais E1 e E2 sugerem que as alterações em termos de conteúdo mineral, devido ao processo de cárie poderão modificar a distribuição de chumbo após a erupção dentária. No entanto, como o chumbo foi observado no 
interior do esmalte, outra possibilidade (aqui citada apenas como especulação) precisa ser explorada, ou seja, o fato de que o chumbo também possa deixar o esmalte dentário durante 0 processo de formação da lesão cariosa. Esta especulação se baseia no fato do chumbo estar presente em concentrações muito altas (da ordem de centenas a milhares de ppm) na superfície do esmalte. Assim, o pH ácido e a desmineralização possivelmente favoreceriam que o chumbo presente em grandes quantidades na superfície do esmalte deixasse este e passasse à saliva, sendo assim deglutido. Esta seria uma nova via de exposição a chumbo a partir de uma fonte endógena: o esmalte dentário (Costa de Almeida et al., 2009). 


\section{CONCLUSÃO}

Em conclusão, este estudo demonstra pela primeira vez, que, sob condições de cárie in vitro, o chumbo se acumula na superfície do esmalte dentário nas condições empregadas para o grupo controle e na concentração de $30 \mu \mathrm{g} / \mathrm{L}$ de acetato de chumbo testada.

Nenhuma significância na incorporação de chumbo foi detectada em profundidades superiores a $50 \mu \mathrm{m}$. A exposição do esmalte a $300 \mu \mathrm{g} / \mathrm{L}$ de acetato de chumbo nas soluções de desmineralização e remineralização resultou na incorporação de chumbo nas camadas mais profundas do esmalte, atingindo uma profundidade de até $200 \mu \mathrm{m}$. 
7 REFERÊNCIAS 


\section{REFERÊNCIAS}

ACCLPP. Low Level Lead Exposure Harms Children: A Renewed Call for Primary Prevention. Report of the Advisory Committee on Childhood Lead Poisoning Prevention of the Centers for Disease Control and Prevention. Jan, 2012.

Argenta RMO, Tabchoury CPM, Cury JC. A modified pH cycling model to evaluate fluoride effect on enamel demineralization. Braz Oral Res. 2003 Jul-Sep;17(3):241-246.

Arora M, Chan SW, Ryan CG, Kennedy BJ, Walker DM. Spatial distribution of lead in enamel and coronal dentine of Wistar rats. Biol Trace Elem Res. 2005 Summer;105(1-3):159-170.

Barbosa F Jr, Corrêa Rodrigues MH, Buzalaf MR, Krug FJ, Gerlach RF, Tanus-Santos JE. Evaluation of the use of salivary lead levels as a surrogate of blood lead or plasma lead levels in lead exposed subjects. Arch Toxicol 2006 Oct;80(10):633-637.

Bergdahl IA, Vahter M, Counter SA, Schütz A, Buchanan LH, Ortega F, Laurell G, Skerfving $S$. Lead in plasma and whole blood from leadexposed children. Environ Res 1999 Jan;80(1):25-33.

Brudevold F, Steadman LT. The distribution of lead in human enamel. J Dent Res. 1956 Jun;35(3):430-437.

Brudevold F, Aasenden R, Srinivasian BN, Bakhos Y. Lead in enamel and saliva, dental caries and the use of enamel biopsies for measuring past exposure to lead. $\mathrm{J}$ Dent Res. 1977 Oct;56(10):1165-1171.

Cleymaet R, Bottenberg P, Retief DH, Slop D, Michotte $\mathrm{Y}$, Coomans D. In vivo use of a dual acid etch biopsy for the evaluation of lead profiles in human surface enamel. Caries Res. 1991a;25(4):256-263.

Cleymaet R, Quartier R, Slop D, Retief DH, Smeyers-Verbeke J, Coomans D. Model for assessment of lead content in human surface enamel. J Toxicol Environ Health. 1991b Feb;32(2):111-127.

Cleymaet R, Bottenberg P, Slop D, Clara R, Coomans D. Study of lead and cadmium content of surface enamel of schoolchildren from an industrial area in Belgium. Community Dent Oral Epidemiol. 1991c Apr;19(2):107-111. 
Cleymaet R, Retief DH, Quartier E, Slop D, Coomans D, Michotte Y. A comparative study of the lead and cadmium content of surface enamel of Belgian and Kenyan children. Sci Total Environ. 1991d May;104(3):175-189.

Cleymaet R, Collys K, Retief DH, Michotte Y, Slop D,Taghon E, Maex W,Coomans D. Relation between lead in surface tooth enamel, blood, and saliva from children residing in the vicinity of a non-ferrous metal plant in Belgium. $\mathrm{Br} \mathrm{J}$ Ind Med. 1991e Oct;48(10):702-709.

Costa de Almeida GR, Saraiva MCP, Barbosa FJr, Krug FJ, Cury JA, Sousa MLR, Buzalaf MAR, Gerlach RF. Lead contents in the surface enamel of deciduous teeth sampled in vivo from children in uncontaminated and in lead-contaminated areas. Environ Res. 2007 Jul;104(3):337-345.

Costa de Almeida GR, de Freitas CU, Barbosa FJr, Tanus-Santos JE, Gerlach RF. Lead in saliva from lead-exposed and unexposed children. Sci Total Environ. 2009 Feb;407(5):1547-1550.

Costa de Almeida GR, Tavares CFF, de Souza AM, de Sousa TS, Funayama CAR, Barbosa F Jr, Tanus-Santos JE, Gerlach RF. Whole blood, serum, and saliva lead concentrations in 6- to 8-year-old children. Sci Total Environ. 2010 Mar;408(7):15511556.

Costa de Almeida GR, de Souza Guerra C, de Angelo Souza Leite G, Antonio RC, Barbosa F Jr, Tanus-Santos JE, Gerlach RF. Lead contents in the surface enamel of primary and permanent teeth, whole blood, serum, and saliva of 6- to 8-year-old children. Sci Total Environ. 2011Apr;409(10):1799-1805.

Cury JA, Rebelo MAB, Del Bel Cury AA, Derbyshire MTVC, Tabchoury CPM. Biochemical composition and cariogenicity of dental plaque formed in the presence of sucrose or glucose and fructose. Caries Res. 2000 Nov-Dec;34(6):491-497.

De Almeida GR, de Souza Guerra C, Tanus-Santos JE, Barbosa F Jr, Gerlach RF. A plateau detected in lead accumulation in subsurface deciduous enamel from individuals exposed to lead may be useful to identify children and regions exposed to higher levels of lead. Environ Res. 2008 Jun;107(2):264-270.

De Capitani EM. Metabolismo do chumbo em crianças e adultos. Medicina (Ribeirão Preto) 2009 Jul-Sep;42(3):278-286. 
De Capitani EM, Paoliello MMB, Costa de Almeida GR. Fontes de exposição humana ao chumbo no Brasil. Medicina (Ribeirão Preto) 2009 Jul-Sep;42(3):311318.

De Souza Guerra C, Fernanda Gerlach R, Graciele Villela Pinto N, Coutinho Cardoso S, Moreira S, Pereira de Almeida A, Teixeira Alves Peixoto I, Henrique Meloni C, Lemos Mota C, Fernando de Oliveira L, Braz D, Cely Barroso R. X-ray fluorescence with Synchrotron radiation to elemental analysis of lead and calcium content of primary teeth. Appl Radiat Isot. 2010 Jan;68(1):71-75.

Gerlach RF, Cury JA, Krug FJ, Line SRP. Effect of lead on dental enamel formation. Toxicology. 2002 Jun;175(1-3):27-34.

Gomes VE, Sousa MLR, Saraiva MCP, Barbosa FJr, Krug FJ, Cury JA, Gerlach RF. In vivo studies on lead content of enamel surface of deciduous teeth of preschool children. Sci Total Environ. 2004 Mar;320(1):25-35.

Grant LD. Lead and Compounds. In: John Wiley \&Sons, Inc. Environmental Toxicants. 3a. Ed. New Jersey: Morton Lippmann; 2009. p. 757-809.

Needleman HL, Gunnoe C, Leviton A, Reed R, Peresie H, Maher C, Barret P. Deficits in psychologic and classroom performance of children with elevated dentine lead levels. N Engl J Med. 1979 Mar;300(13):689-695.

Needleman HL, Schell A, Bellinger D, Leviton A, Allred EN. The long-term effects of exposure to low doses of lead in childhood. An 11-year follow-up report. $\mathrm{N} \mathrm{Engl} \mathrm{J}$ Med. 1990 Jan;322(2):83-88.

Nanci A. Ten Cate Histologia Oral: desenvolvimento, estruturação e função. Santos CWN, Barroso DRC, Oliveira DP, Fonseca DR, Moritz GC, Caracas HCPM, Pasolini IJ, Silva LM, Nascimento RM, Robaina TF, tradutores. 7aㅡ ed. Rio de Janeiro: Elsevier; 2008. 414 p.

Purchase NG, Fergusson JE. Lead in teeth: the influence of the tooth type and the sample within a tooth on lead levels. Sci Total Environ. 1986 Jul;52(3):239-250.

Queiroz CS, Hara AT, Paes Leme AF, Cury JA. pH-cycling models to evaluate the effect of low fluoride dentifrice on enamel de- and remineralization. Braz Dent J. 2008; 19(1): 21-27. 
Rentz H, Schaefer V, Duschner H, Radlanski RJ. Incremental lines in root cementum of human teeth: an approach to their ultrastructural nature by microscopy. Adv Dent Res. 1997 Nov;11(4):472-477.

Rosner D, Markowitz G, Lanphear B. J. Lockhart Gibson and the discovery of the impact of lead pigments on children's health: a review of a century of knowledge. Public Health Rep. 2005 May-Jun;120(3):296-300.

Simmer JP, Hu JC. Dental enamel formation and its impact on clinical dentistry. J Dent Educ. 2001 Sep;65(9):896-905.

Smith CE. Cellular and chemical events during enamel maturation. Crit Rev Oral Biol Med. 1998;9(2):128-161.

Weatherell JA, Hallsworth AS, Robinson C. The effect of tooth wear on the distribution of fluoride in the enamel surface of human teeth. Arch Oral Biol 1973 Sep;18(9):1175-1189. 
ANEXO 


\title{
ANEXO
}

\section{ANEXO A}

Artigo publicado

Caries Research

\section{Original Paper}

\section{Lead Deposition in Bovine Enamel during a pH-Cycling Regimen Simulating the Caries Process}

\author{
G.F. Molina ${ }^{a}$ G.R. Costa de Almeida ${ }^{a}$ C. de Souza Guerra ${ }^{b} \quad$ J.A. Cury ${ }^{b}$ \\ A.P. de Almeidac ${ }^{c}$ R.C. Barroso ${ }^{d}$ R.F. Gerlach ${ }^{a}$ \\ a Universidade de São Paulo, FORP/USP and buniversidade Estadual de Campinas, FOP/UNICAMP, São Paulo, \\ 'Laboratório de Instrumentação Nuclear, COPPE/UFRJ, and dUniversidade do Estado do Rio de Janeiro, IF/UERJ, \\ Rio de Janeiro, Brasil
}

